\title{
Probable drug-induced liver injury with kedaling tablets : A case report and systematic literature review
}

\author{
jiabing wang ${ }^{1}$ \\ ${ }^{1}$ Affiliation not available
}

September 11, 2020

\begin{abstract}
Kedaling tablets is an extract of with Corydalis yanhusuo W.T. Wang and used to coronary heart disease, angina pectoris, myocardial infarction with no previously well documented hepatotoxicity. We report the first case of kedaling tablets-related drug-induced liver injury (DILI), which highlights the need for clinician awareness regarding potential hepatotoxicity of kedaling tablets, particularly among patients with pre-existing liver disease.
\end{abstract}

Probable drug-induced liver injury with kedaling tablets : A case report and systematic literature review Jiabing Wang ${ }^{*}$

Department of pharmacy, Taizhou Municipal Hospital, Zhejiang Taizhou 318000, China

${ }^{*}$ Correspondence author

E-mail address: wangjiabing9205@163.com

Abstract

Kedaling tablets is an extract of with Corydalis yanhusuo W.T. Wang and used to coronary heart disease, angina pectoris, myocardial infarction with no previously well documented hepatotoxicity. We report the first case of kedaling tablets-related drug-induced liver injury (DILI), which highlights the need for clinician awareness regarding potential hepatotoxicity of kedaling tablets, particularly among patients with pre-existing liver disease.

Key words:kedaling tablets;drug-induced liver injury;traditional Chinese medicine

\section{Background:}

Traditional Chinese medicine (TCM) becomes increasingly used in clinic because of the unique efficacy [1]. However, TCM also is one of the leading causes of drug-induced liver injury (DILI), which has been highly raised. TCM account for approximately $26.81 \%$ of all DILI from 2012 to 2014 at 308 medical centers in mainland China $[2,3]$. Corydalis yanhusuo W.T. Wang (YHS) is a well-known Chinese flowering herbal plant commonly used for centuries in functional food and TCM [4]. kedaling tablets is an extract of YHS, the main active components are dehydrocorydaline, which has been approved for the treatment of coronary heart disease, angina pectoris, myocardial infarction. kedaling tablets have been extremely well tolerated by most patients, drug-induced liver injury has been infrequently described.

\section{Case Presentation:}

We first described herein a case of long-term drug-induced liver injury attributable to kedaling tablets. A patient was a 64 -year-old man with more than 30 years hypertension and more than 1 year coronary disease. 
The patient has an alcohol history of $25 \mathrm{ml}$ of white spirit per day, on average, but there was no known family history of liver disease. He was referred to our outpatient clinic on May 24, 2017 due to hypertension and coronary disease, and he treated with amlodipine/ diltiazem/rabeprazole sodium/heart-protecting musk pill/simvastatin/clopidogrel/ irbesartan and his liver enzymes have been stable. Until August 20, 2018, he add flunarizine/betahistine/kedaling tablets, his liver enzymes were start changed. Over the next 1 month, his alanine transaminase (ALT), aspartate transaminase (AST), gamma-glutamyltransferase (GGT) and alkaline phosphatase (ALP) significantly increased to 382, 178, 90 and 57 IU/L, respectively. An abdominal CT scan did demonstrate fatty liver. On further testing, a viral serology screen was negative for hepatitis A (HAV) IgM, hepatitis C (HCV) IgM and HBc IgM, however, anti-HBs was noted to be weakly positive. Moreover, Anti-liver-kidney-microsomal, antimitochondrial, anti-smooth muscle and anti-centromere antibodies were all negative. After two week of liver protection treatment, his liver enzymes decreased to returned to baseline and he was discharged. His anti-hypertension and anti-coronary heart disease were continued with amlodipine/diltiazem/rabeprazole sodium/ heart-protecting musk pill/simvastatin /clopidogrel/irbesartan/kedaling tablets, however, his ALT, AST, GGT and ALP rebounded to 864, 440, 389 and 93 IU/L for 1 month later. He was re-admission, kedaling tablets was discontinued on the first day, while other drugs were continued. The results of viral serology screen are similar to those of last time. After around one year of follow-up without kedaling tablets, liver enzymes remained normal. Unfortunately, On October 29, 2019, he switched to kedaling tablets, and his ALT, AST, GGT and ALP elevated at 716, 277, 148 and $93 \mathrm{IU} / \mathrm{L} 1$ month later, which suggested that kedaling tablets probably account for DILI. The patient was instructed never to re-challenge himself with kedaling tablets and to report it as a medication adverse reaction in the future. Thus, kedaling tablets was discontinued and liver enzymes returned to baseline and have remained stable until now.

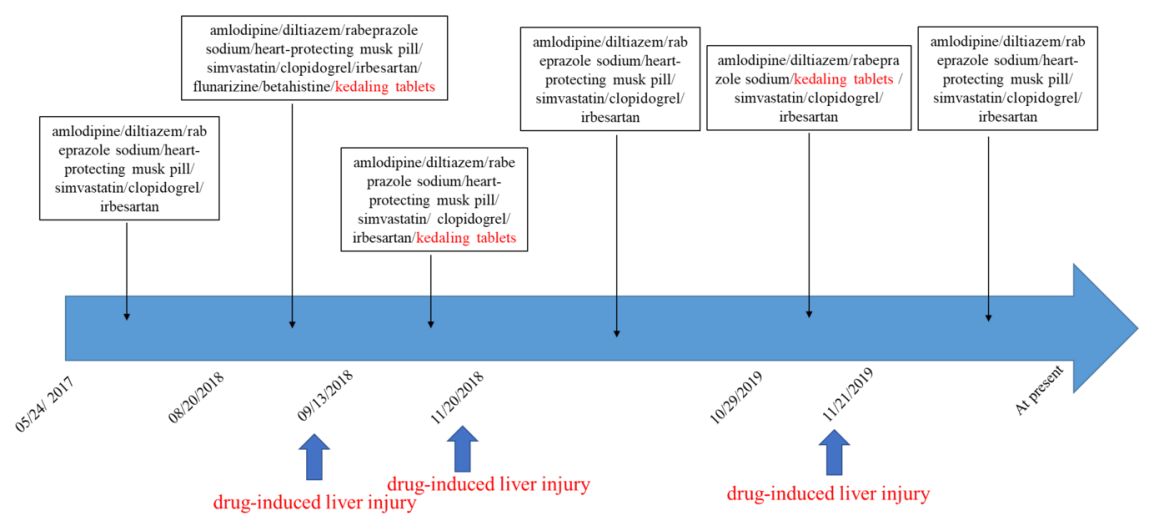

Figure 1. The timeline of the drugs treatment in the case. 


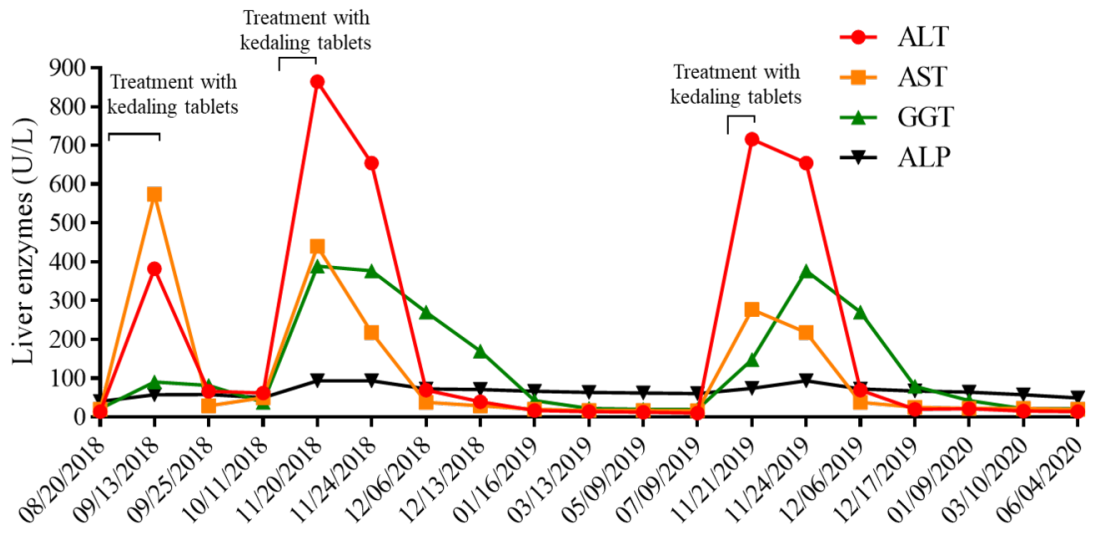

Figure 2. Evolution of AST, ALT, GGT and ALP levels with kedaling tablets exposure and discontinuation.

Table1. The changes of liver enzyme levels before and after taking Ketaling tablets

\begin{tabular}{lllll}
\hline Liver enzymes & ALT (IU/L) & AST (IU/L) & GCT (IU/L) & ALP (IU/L) \\
\hline Before using the medicine & $56.67 \pm 15.69$ & $39.00 \pm 10.54$ & $159.33 \pm 116.37$ & $64.33 \pm 12.42$ \\
After using the medicine & $654.00 \pm 246.91$ & $298.33 \pm 132.30$ & $654 \pm 246.91$ & $74.67 \pm 18.01$ \\
P Value & $¡ 0.01$ & $i 0.01$ & $i 0.05$ & $\iota 0.05$ \\
t-Test & 4.039 & 3.150 & 0.507 & 0.228 \\
\hline
\end{tabular}

Discussion and conclusion

Hepatic injury caused by both prescription and non-prescription, is termed drug-induced liver injury (DILI) [5]. DILI ranges in severity from mild, asymptomatic, elevations in liver enzymes to severe hepatic injury culminating in acute liver failure resulting and death [5]. Up to now, there are currently just under a thousand drugs that have been suspected of causing DILI clinically. Research showed that TCM or herbal and dietary supplements (HDS) are one of the leading causes of drug-induced liver injury (DILI) [6].

Kedaling tablets is an extract of with Corydalis yanhusuo W.T. Wang and used to coronary heart disease, angina pectoris, myocardial infarction with no previously well documented hepatotoxicity. We report a case of a 64-year-old man who had hypertension, coronary disease and fatty liver. After 1 year on related antihypertensive and anti-coronary heart disease treatments, his liver enzymes in standard conditions. But he was initially treated with kedaling tablets and other drugs, and approximately one month later, his liver enzymes were elevated, but doctors did not know the DILI was caused by the kedaling tablets. Over the next month, the patient still experienced serious DILI after being on kedaling tablets and other drugs. Doctors analyzed the patient's medication for nearly a year and suspected that DILI may relate to kedaling tablets therapy. Thus, during a follow-up of 12 months, his liver enzymes remained normal without kedaling tablets, though he had fatty liver. However, when he took kedaling tablets again, his liver enzymes rebounded. More importantly, after 8 months of follow-up without kedaling tablets, liver enzymes remained normal. In our patient's case, we attributed liver injury to kedaling tablets on the basis of the facts that drug exposure preceded the onset of liver injury, though the patient suffered from fatty liver, cessation of the drug led to improvement in liver enzymes, and symptoms recurred rapidly when the patient was re-challenged (Figure 1 and 2). Extensive testing for other causes of acute liver injury was negative and the patient did not endorse signs, symptoms or imaging findings suggestive of outflow obstruction, hepatic ischemia or biliary obstruction. Although the patient was discovered to be anti-HBs positive and fatty liver, these were very unlikely to be an acute infection. DILI may be classified into three categories based upon the pattern of 
liver injury observed, including acute hepatocellular injury, cholestatic injury and mixed liver injury [7]. Hepatocellular injury is due to substantial damage to the hepatocytes and is characterized by elevations in the serum AST and ALT levels, with lesser elevations in the ALP level [8]. In the case of our patient, he demonstrated a predominantly hepatocellular injury that occurred with substantially elevated AST and ALT levels and a mildly elevated ALP level (Table1).

It is found that the toxicity of TCM generally depend on their basic substances [6]. Because dehydrocorydaline is the main active ingredient in kedaling tablets, we suspected that the mechanism of kedaling tablets-induced DILI may relate to dehydrocorydaline in the case report presented here. We searched the PubMed database to identify literature with the dehydrocorydaline-induced DILI, but there are not publications. But studies have reported that dehydrocorydaline could inhibit the tumorigenesis of breast cancer MDA-MB-231 cells through downregulating cell proliferation, anti-apoptosis, metastasis-associated proteins CDK1, CCND1, BCL2 and metastasis-associated proteins MMP2 and MMP9, and upregulating the expression of proapoptotic proteins caspase $3 / 8 / 9$, suggesting dehydrocorydaline is a multi-target cytotoxic drug and it has the potential to damage liver cells [9]. Dehydrocorydaline also could inhibit cell proliferation, migration and invasion via suppressing MEK1/2-ERK1/2 cascade in melanoma [10]. Besides, the structure of dehydrocorydaline is similar to that of the hepatotoxic pyrrodines alkaloids. Therefore, the case of DILI may relate to dehydrocorydaline therapy in our study.

In summary, this patient presented DILI with substantially elevated AST and ALT levels following introduction of kedaling tablets. To our knowledge, this is the first case of documented hepatotoxicity induced by kedaling tablets and the exact mechanisms of how kedaling tablets causes liver injury require further research.

Conflicts of Interests

The authors declare that there are no competing interests

Acknowledgements

None

References

1. Liu C, Fan H, Li Y, Xiao X. Research advances on hepatotoxicity of herbal medicines in China. BioMed research international. 2016;2016:7150391.

2. Byeon JH, Kil JH, Ahn YC, Son CG. Systematic review of published data on herb induced liver injury. Journal of ethnopharmacology. 2019;233:190-6.

3. Shen T, Liu Y, Shang J, Xie Q, Li J, Yan M, et al. Incidence and etiology of drug-induced liver injury in mainland China. Gastroenterology. 2019;156:2230-41 e11.

4. Wan L, Zhao Y, Zhang Q, Gao G, Zhang S, Gao Y, et al. Alkaloid extract of Corydalis yanhusuo inhibits angiogenesis via targeting vascular endothelial growth factor receptor signaling. BMC complementary and alternative medicine. 2019;19:359.

5. Norman BH. Drug Induced Liver Injury (DILI). Mechanisms and medicinal chemistry avoidance/mitigation strategies. Journal of medicinal chemistry. 2020.

6. Pan X, Zhou J, Chen Y, Xie X, Rao C, Liang J, et al. Classification, hepatotoxic mechanisms, and targets of the risk ingredients in traditional Chinese medicine-induced liver injury. Toxicology letters. 2020;323:48-56.

7. Chalasani N, Fontana RJ, Bonkovsky HL, Watkins PB, Davern T, Serrano J, et al. Causes, clinical features, and outcomes from a prospective study of drug-induced liver injury in the United States. Gastroenterology. 2008;135:1924-34, 34 e1-4.

8. Ellison CA, Blackwell SB. Acute Hepatocellular injury associated with azithromycin. Journal of pharmacy practice. 2020:897190019894428.

9. Huang Y, Huang H, Wang S, Chen F, Zheng G. Dehydrocorydaline inhibits the tumorigenesis of breast cancer MDAMB231 cells. Molecular medicine reports. 2020;22:43-50. 
10. Hu H, Dong Z, Wang X, Bai L, Lei Q, Yang J, et al. Dehydrocorydaline inhibits cell proliferation, migration and invasion via suppressing MEK1/2-ERK1/2 cascade in melanoma. OncoTargets and therapy. 2019;12:5163-75. 